\title{
HISTÓRIA DA PROMOÇÃO DA SAÚDE E A "CARTA DE OTTAWA DESCRÍTA NA INTEGRA"
}

\author{
Cassio Hartmann ${ }^{1}$ \\ Gabriel César Dias Lopes² \\ Fábio da Silva Ferreira Vieira ${ }^{3}$ \\ Bensson V Samuel ${ }^{4}$
}

\section{RESUMO}

No início da década de 1940, iniciou-se a discussão sobre saúde e com o passar dos anos, esse assunto foi tomando uma proporção gigantesca em todo o planeta e por fim este artigo aborda a história, o conceito de promoção da saúde, a evolução das conferências internacionais e a carta de Ottawa que é considerada peça chave para as diretrizes em busca da promoção da saúde, principalmente com novas políticas públicas e com a disseminação de pesquisas com seres humanos visando melhorias na saúde e na qualidade de vida. Conclui-se que a Carta de Ottawa ainda permanece como peça central de direcionamento da estratégia de promoção à saúde em todo o mundo. Essa peça chave tem orientado as demais conferências, principalmente quando enfatiza a dimensão social e a importância das cinco estratégias fundamentais para se alcançar plena saúde: política pública, ambiente saudável, reforço da ação comunitária, criação de habilidades pessoais e reorientação do serviço de saúde. São condições imprescindíveis e os recursos fundamentais para a saúde são: Paz Habitação - Educação - Alimentação - Renda - ecossistema estável - recursos sustentáveis - justiça social e equidade. O incremento nas condições de saúde requer uma base sólida nestes pré-requisitos básicos para a promoção da saúde.

Palavras-chaves: História; Promoção da Saúde; Carta Ottawa.

\footnotetext{
${ }^{1}$ Professor de Educação Física SEM FRONTEIRAS DA FIEP-BRASIL / Delegado Adjunto Nacional da Federação Internacional de Educação Física FIEP / Secretario e Imortal da ABEF - Academia Brasileira de Educação Física / Conselheiro CREF 19AL / Professor de Educação Física do Instituto Federal de Alagoas/IFAL e doutorando em saúde coletiva com ênfase em Educação Física E-mail: cassiohartmann04@gmail.com

${ }^{2}$ Prof. Dr. Gabriel C. D. Lopes, PhD Professor e Orientador Doutor em Educação / PhD em Psicanálise Clínica Presidente da LUI - Logos University Int.Professor / Membro Imortal da ABEF - Academia Brasileira de Educação Física - E-mail: president@unilogos.education
} 
${ }^{3}$ Professor Co-orientador / Doutor em Ciências do Movimento Humano / Mestre em Educação Física / Especialista em Fisiologia do Exercício / Delegado Adjunto da Federação Internacional de Educação Física FIEP-PR. E-mail: vieira.fsf@gmail.com

${ }^{4}$ Professor de Ciências e Médico / Bacharel em Medicina Poznan University of Medical Science, Poland/ Bacharel em Ciências Médicas e Laboratoriais (Cito-Tecnologia) University of Connecticut, Storrs, CT, USA / Especialista em Clínica Geral Queen Mary University / Especialista em Urgência e Emergência Medvarsity-Apollo Hospital / Doutorado em Liderança e Gestão Estratégica London School of Internation Business / Doutorado PhD: Pan-American University - Health Care Management / Doutorado PhD: Swiss Open University in Economics. E-mail: contact@unilogos.org

\section{ABSTRACT}

At the beginning of the 1940s, the discussion on health began and over the years, this subject has taken on a gigantic proportion across the planet and finally this article addresses the history, the concept of health promotion, evolution the international conferences and the Ottawa charter, which is considered a key piece of guidelines in search of health promotion, mainly with new public policies and with the dissemination of research with human beings aiming at improvements in health and quality of life. It is concluded that the Ottawa Charter still remains as a central piece of direction for the health promotion strategy worldwide. This key piece has guided other conferences, especially when it emphasizes the social dimension and the importance of the five fundamental strategies to achieve full health: public policy, healthy environment, reinforcement of community action, creation of personal skills and reorientation of the health service. These are essential conditions and the fundamental resources for health are: Peace - Housing - Education - Food - Income - stable ecosystem sustainable resources - social justice and equity. The increase in health conditions requires a solid foundation in these basic prerequisites for health promotion.

Keywords: History, Health Promotion, Ottawa Charter.

\section{RESUMEN}

A principios de la década de 1940, comenzó la discusión sobre la salud y, a lo largo de los años, este tema ha adquirido una proporción gigantesca en todo el planeta y finalmente este artículo aborda la historia, el concepto de promoción de la salud, la evolución las conferencias internacionales y la carta de Ottawa, que se considera una pieza clave de las directrices en busca de la promoción de la salud, principalmente con nuevas políticas públicas y con la difusión de investigaciones con seres humanos para mejorar la salud y la calidad de vida. Se concluye que la Carta de Ottawa sigue siendo una pieza central de dirección para la estrategia de promoción de la salud en todo el mundo. Esta pieza clave ha guiado otras conferencias, especialmente cuando enfatiza la dimensión social y la importancia de las cinco estrategias fundamentales para lograr la salud plena: políticas públicas, ambiente saludable, refuerzo de la acción comunitaria, creación de habilidades personales y reorientación del servicio de salud. Estas son condiciones esenciales y los recursos fundamentales para la salud son: Paz - Vivienda - Educación - Alimentos - Ingresos - ecosistema estable - recursos 
sostenibles - justicia social y equidad. El aumento de las condiciones de salud requiere una base sólida en estos prerrequisitos básicos para la promoción de la salud.

Palabras clave: Historia, Promoción de la Salud, Carta de Ottawa.

\section{INTRODUÇÃO}

A Promoção da Saúde é um campo do conhecimento ainda recente na história da Saúde Pública e ao mesmo tempo encontra-se em plena evolução com várias pesquisas que tentam desvendar suas inovações e aplicações através de vários experimentos em saúde nas mais variadas populações (MELLO et al., 2014; HARTMANN et al., 2020).

Ao longo dos anos 70 e 80, as evidências da associação entre condições de vida, prosperidade e bom nível educacional se acumularam (TERRIS, 1992; MENDES, 1996). Dessa forma foram criadas as condições para a organização da I Conferência Internacional em Promoção de Saúde, em 1996, em Ottawa no Canadá, patrocinada pela OMS - Organização Mundial da Saúde, Ministério da Saúde e Bem Estar do Canadá e Associação Canadense de Saúde Pública (WHO, 1986)

\footnotetext{
Segundo (XAVIER, 2017) a Carta de Ottawa aponta a Promoção da Saúde "para além dos cuidados de saúde" com "a adoção de políticas públicas saudáveis nos setores que não estão diretamente ligados à saúde". A Carta de Ottawa estabeleceu o desafio de uma mudança para a nova saúde pública, reafirmando a justiça social e a equidade como pré-requisitos para a saúde, advocacia e mediação como processos para sua realização.
}

Este artigo está dividido em introdução e mais três subtópicos. No primeiro é descrita a história e o conceito de promoção da saúde, citado pelos primeiros pesquisadores, com por exemplo Sigerist (1946), Leavell e Clark (1965) e Lalonde (1974) e procurando seguir uma sequência cronológica sobre o tema abordado. No segundo aborda-se sobre a história da carta de Ottawa e a evolução da promoção da saúde, embora, tenha sido escrita no final do século $X X$, tem sido de fundamental importância e referência para os profissionais da área de saúde na atualidade.

No terceiro subtópico a carta de Ottawa na sua integra e que completará trinta e quatro (34) anos, no mês de novembro de 2020 e que é um grande referencial no século XXI para todo o mundo.

De acordo com Xavier (2017) a referida pesquisa é um estudo analítico, com abordagem qualitativa que adota como instrumentos para a coleta dos dados a 
pesquisa bibliográfica e a pesquisa documental, ambas realizadas com base em roteiro previamente estabelecido conforme orientações de Severino (2016).

Portanto faz-se necessário conhecer a história, o conceito da promoção da saúde e a Carta de Ottawa.

\section{HISTÓRIA DO CONCEITO DA PROMOÇÃO DA SAÚDE}

Segundo Becker (2001) nas últimas décadas, tem-se assistido e acompanhado um crescimento imenso no campo de promoção da saúde, que vem desenvolvendo através de várias pesquisas e consequentemente com várias concepções, interpretações e práticas de saúde pública e coletiva no atual século XXI.

Em 1943, a Medicina Social inglesa se preocupava com a epidemiologia das doenças crônicas prevalentes, como a úlcera péptica, as doenças cardiovasculares, o câncer e os traumas acidentais. Considerando suas relações com as condições sociais e ocupacionais, afirmava que elas deveriam ser, em maior ou menor grau, de prevenção (TERRIS, 1992).

Sigerist (1946) foi um dos primeiros autores a utilizar a expressão "promoção da saúde" ao definir as quatro tarefas primordiais da medicina, a saber:

1ำ a promoção da saúde;

2a prevenção de doenças:

3ำ a recuperação do enfermo;

4ำ a reabilitação.

Importante ressaltar que, ao listar os principais pontos de um programa nacional de saúde, Sigerist (1946) destacou a educação gratuita universal, boas condições de vida e trabalho, oportunidades para descanso e recreação como as três mais importantes, deixando a atenção médica na quarta posição.

Leavell e Clark (1965) ao desenvolverem o modelo de história natural de doença e seus três níveis de prevenção, incluem a promoção da saúde na prevenção primária, como medida destinada a melhorar a saúde e o bem estar geral. As atividades a serem realizadas para promover a saúde incluiriam a boa nutrição, 0 atendimento às necessidades afetivas, educação sexual, orientação pré-nupcial e parental, boas condições de moradia, trabalho e lazer, além de exames periódicos e educação para a saúde. 
Lalonde (1974) afirma que, até aquele momento, a maioria dos esforços da sociedade para melhorar a saúde, e a maior parte dos gastos em saúde, se concentravam na organização do cuidado médico, apesar disso, quando identificavam-se as causas principais de adoecimento e morte no Canadá, verificavase que a sua origem estava nos três outros componentes do conceito de campo, a saber: a biologia humana, o meio ambiente e o estilo de vida.

Ainda para Lalonde (1974), o marco inicial da moderna Promoção da Saúde é no Canadá, e é considerado por Terris (1992) "a primeira declaração teórica e abrangente na Saúde Pública como resultado dos desconhecimentos de epidemiologia de doenças não infecciosas". Utilizando o modelo de "Campo de Saúde", proposto por Laframboise em 1973 (FERRAZ, 1996; CARVALHO, 1996), o documento afirma que a saúde é determinada por um conjunto de fatores agrupáveis em quatro categorias:

- Biologia humana - que envolve todos os fatos que se manifestam como consequência da constituição orgânica do indivíduo, incluindo sua herança genética e seus processos de maturação;

- Meio ambiente - agrupa os fatores externos ao organismo, em suas dimensões física e social, sobre os quais o indivíduo exerce pouco ou nenhum controle;

- Estilos de vida - o conjunto das decisões que o indivíduo toma a respeito da sua saúde, no que se refere por exemplo a suas atividades de lazer e alimentação, estando, portanto, parcialmente sob seu controle.

- Organização da atenção à saúde - disponibilidade, quantidade e qualidade dos recursos destinados aos cuidados com a saúde.

Associação da promoção da saúde, com a atuação sobre determinantes do meio ambiente e dos estilos de vida surgiu, com a "segunda revolução epidemiológica": o movimento de prevenção das doenças crônicas, segundo Terris (1992).

Uma melhor nutrição, melhorias no saneamento básico, e modificações nas condutas da reprodução humana (com menos o número de filhos por família), há muito tempo são reconhecidas como os principais fatores responsáveis pela redução da mortalidade na Inglaterra e no País de Gales no século XIX e início do século XX. As 
intervenções médicas, como as vacinações e o advento da antibioticoterapia tiveram influência mais tardia e menos importante (MACKEOWN, 1976).

A influência da Medicina Social inglesa, se fez sentir profundamente no Canadá, refletindo-se na consagração dos princípios de universalização de assistência médica e na centralidade da prevenção e da promoção da saúde no sistema de saúde canadense. A constatação do papel fundamental dos determinantes gerais sobre as condições de saúde, que vem a qualificar a moderna promoção de saúde, deve muito à atuação da saúde pública canadense, e à sua posterior influência internacional (FERRAZ, 1996).

Segundo Hartmann et al., (2020) a atuação nas ações de promoção da saúde estão mediante práticas corporais, atividades físicas e de lazer na prevenção primária, secundária e terciária no SUS - Sistema único de Saúde e no setor privado.

Para Buss (1998), a Carta de Ottawa (1986) "assume que a equidade em saúde é um dos focos da promoção da saúde, cujas ações objetivam reduzir as diferenças no estado de saúde da população, e no acesso a recursos diversos para uma vida mais saudável".

Para Ferraz (1996), a Carta de Ottawa "significou a ampliação da concepção de promoção da saúde, incorporando a importância e o impacto das dimensões socioeconômicas, políticas e culturais sobre as condições de saúde. Houve um reconhecimento de que a promoção de saúde não concerne exclusivamente ao setor saúde, mas ao contrário, ela se constitui numa atividade essencialmente intersetorial".

Para Carvalho (1996), o conceito de promoção da saúde passa a ser a espinha dorsal da nova saúde pública e é definido pela primeira vez em termos de políticas e estratégias, representando "um avanço em relação à retórica genérica da Conferência de Alma-Ata (1977), que estabelecera a consigna 'saúde para todos até o ano 2000', através da expansão da atenção primária".

Ferraz (1996), Buss (1998) e Mendes (1998) afirmam que este movimento, origina ou fortalece conceitos fundamentais do moderno mundo do campo sanitário, tais como as cidades saudáveis, os ambientes favoráveis à saúde, a articulação intersetorial pela saúde, vigilância à saúde e as diversas práticas de reorientação de serviços.

A WHO (1986) estabelece pela Carta de Ottawa as estratégias de ação em: 
1) estabelecimento de políticas públicas saudáveis;

2) criação de ambientes favoráveis à saúde;

3) reforço da ação comunitária;

4) desenvolvimento de habilidades pessoais;

5) reorientação dos serviços de saúde.

Na I Conferência Internacional em Promoção da Saúde da OMS, em Ottawa, que reconheceu como pré-requisitos fundamentais para a saúde: "a paz, a educação, a habitação, o poder aquisitivo, um ecossistema estável, e conservação dos recursos naturais e a equidade". A promoção da saúde foi conceituada pela "Carta de Ottawa" - um documento que marcou definitivamente a história da Saúde Pública - como "o processo de capacitação na comunidade para atuar na melhoria da sua qualidade de vida e saúde, incluindo uma maior participação no controle deste processo". A Carta assume que a equidade deve ser um dos focos da promoção da saúde: seu objetivo estabelece-se em reduzir as diferenças no estado de saúde da população, e no acesso a recursos diversos para uma vida mais saudável.

A promoção da saúde não concerne exclusivamente ao setor saúde propriamente dito. Ao contrário, ela é o resultado de ações intersetoriais, agindo nos determinantes gerais da saúde e da qualidade de vida. Cada setor (educação, geração de trabalho e renda, habitação, lazer e cultura, transportes, meio ambiente, assistência social, etc.) deverá ter suas estratégias de atuação, coordenadas por "políticas saudáveis".

O setor saúde propriamente dito tem um importante papel a cumprir neste processo, mas deve ir além da simples provisão de serviços clínicos e assistenciais. Deve apoiar indivíduos e comunidades para uma vida mais saudável, articulando-se com os setores sociais, políticos, econômicos e ambientais.

\section{HISTÓRIA DA CARTA DE OTTAWA E A EVOLUÇÃO DA PROMOÇÃO DA SAÚDE}

A Carta de Ottawa para a Promoção da Saúde é um marco fundamental na história da Saúde Pública, reconheceu como "pré-requisitos fundamentais para a 
saúde: a paz, a educação, a habitação, o poder aquisitivo, um ecossistema estável, e conservação dos recursos naturais e a equidade" (WHO, 1986).

Desde sua elaboração, a Carta de Ottawa, se tornou o principal documento de referência da promoção da saúde em todo o mundo, como comprovam documentos oriundos das diversas conferências internacionais e temas, como mostra o quadro 01.

\section{Quadro 01: Conferências Internacionais}

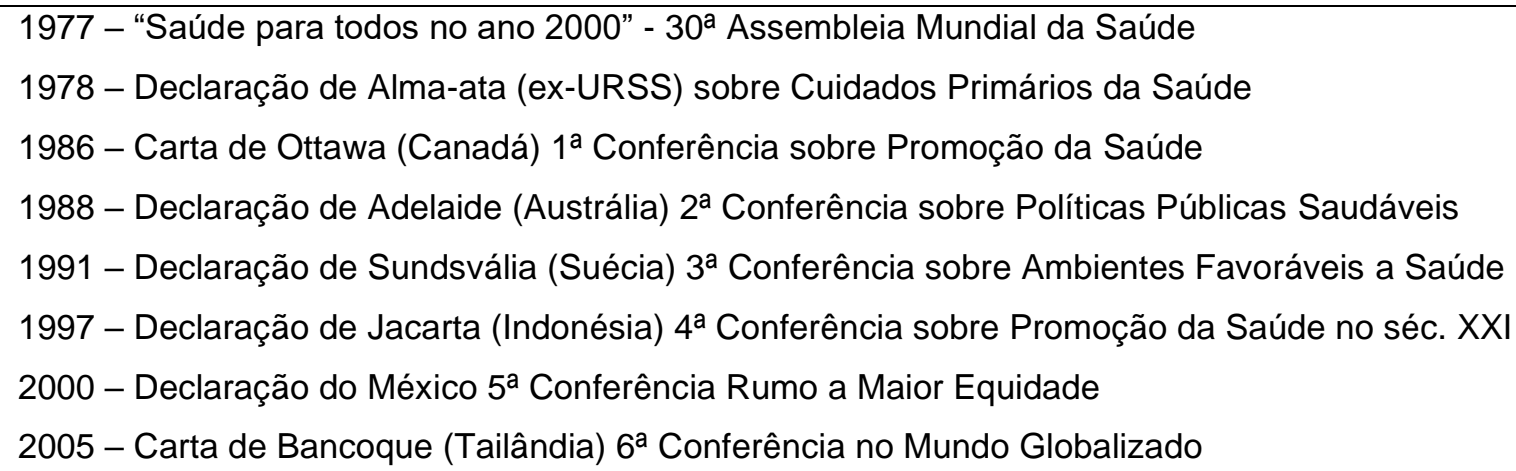

Na América Latina, a Organização Panamericana de Saúde - OPAS - colocoua como prioridade programática, definindo-a como "a soma das ações da população, dos serviços de saúde, das autoridades sanitárias e de outros setores sociais e produtivos, dirigidas para o desenvolvimento de melhores condições de saúde individual e coletiva" (MENDES, 1998).

Com a evolução do movimento da promoção de saúde na América do Norte surge o Movimento Cidades Saudáveis, citado aqui por sua importância na compreensão do alcance e do significado da nova formulação dos conceitos de promoção de saúde. O movimento surgiu em 1984, em Toronto no Canadá, e foi encampado pela OMS a partir de 1986.

A OMS considera uma cidade saudável aquela em que os dirigentes municipais enfatizam a saúde de seus cidadãos numa ótica ampliada de qualidade de vida, de acordo com a proposta de promoção da saúde desenvolvida na Carta de Ottawa.

Esse movimento difundiu-se em inúmeras cidades de todo o mundo, formando redes regionais e globais, e tornou-se o mais difundido projeto estruturante da moderna promoção de saúde, tendo sido também incorporado como estratégia pelo 
movimento de municipalização da saúde no Brasil (FERRAZ, 1993; FERRAZ, 1996; BUSS, 1998; MENDES, 1998).

\title{
4 CARTA DE OTTAWA (PUBLICAÇÃO NA ÍNTEGRA)
}

\author{
1․ CONFERÊNCIA INTERNACIONAL \\ SOBRE PROMOÇÃO DA SAÚDE \\ Ottawa, novembro de 1986
}

A Primeira Conferência Internacional sobre Promoção da Saúde, realizada em Ottawa, Canadá, em novembro de 1986, apresenta neste documento sua Carta de Intenções, que seguramente contribuirá para se atingir Saúde para Todos no Ano 2000 e anos subsequentes.

Esta Conferência foi, antes de tudo, uma resposta às crescentes expectativas por uma nova saúde pública, movimento que vem ocorrendo em todo o mundo. As discussões localizaram principalmente as necessidades em saúde nos países industrializados, embora tenham levado em conta necessidades semelhantes de outras regiões do globo.

As discussões foram baseadas nos progressos alcançados com a Declaração de Alma- Ata para os Cuidados Primários em Saúde, com o documento da OMS sobre Saúde Para Todos, assim como o debate ocorrido na Assembleia Mundial da Saúde sobre as ações intersetoriais necessárias para o setor.

\section{PROMOÇÃO DA SAÚDE}

Promoção da saúde é o nome dado ao processo de capacitação da comunidade para atuar na melhoria de sua qualidade de vida e saúde, incluindo uma maior participação no controle deste processo. Para atingir um estado de completo bem-estar físico, mental e social os indivíduos e grupos devem saber identificar aspirações, satisfazer necessidades e modificar favoravelmente o meio ambiente. A saúde deve ser vista como um recurso para a vida, e não como objetivo de viver. Nesse sentido, a saúde é um conceito positivo, que enfatiza os recursos sociais e 
pessoais, bem como as capacidades físicas. Assim, a promoção da saúde não é responsabilidade exclusiva do setor saúde, e vai para além de um estilo de vida saudável, na direção de um bem-estar global.

\section{PRÉ-REQUISITOS PARA A SAÚDE}

As condições e os recursos fundamentais para a saúde são: Paz - Habitação - Educação - Alimentação - Renda - ecossistema estável - recursos sustentáveis justiça social e equidade. $O$ incremento nas condições de saúde requer uma base sólida nestes pré-requisitos básicos.

\section{DEFESA DE CAUSA}

A saúde é o maior recurso para o desenvolvimento social, econômico e pessoal, assim como uma importante dimensão da qualidade de vida. Fatores políticos, econômicos, sociais, culturais, ambientais, comportamentais e biológicos podem tanto favorecer como prejudicar a saúde. As ações de promoção da saúde objetivam, através da defesa da saúde, fazer com que as condições descritas sejam cada vez mais favoráveis.

\section{CAPACITAÇÃO}

Alcançar a equidade em saúde é um dos focos da promoção da saúde. As ações de promoção da saúde objetivam reduzir as diferenças no estado de saúde da população e assegurar oportunidades e recursos igualitários para capacitar todas as pessoas a realizar completamente seu potencial de saúde. Isto inclui uma base sólida: ambientes favoráveis, acesso à informação, a experiências e habilidades na vida, bem como oportunidades que permitam fazer escolhas por uma vida mais sadia. As pessoas não podem realizar completamente seu potencial de saúde se não forem capazes de controlar os fatores determinantes de sua saúde, o que se aplica igualmente para homens e mulheres. 


\section{MEDIAÇÃO}

Os pré-requisitos e perspectivas para a saúde não são assegurados somente pelo setor saúde. Mais importante, a promoção da saúde demanda uma ação coordenada entre todas as partes envolvidas: governo, setor saúde e outros setores sociais e econômicos, organizações voluntárias e não-governamentais, autoridades locais, indústria e mídia. As pessoas, em todas as esferas da vida, devem envolverse neste processo como indivíduos, famílias e comunidades. Os profissionais e grupos sociais, assim como o pessoal de saúde, têm a responsabilidade maior na mediação entre os diferentes, em relação à saúde, existentes na sociedade.

As estratégias e programas na área da promoção da saúde devem se adaptar às necessidades locais e às possibilidades de cada país e região, bem como levar em conta as diferenças em seus sistemas sociais, culturais e econômicos.

\section{SIGNIFICADO DAS AÇÕES DE PROMOÇÃO DA SAÚDE: CONSTRUINDO POLÍTICAS PÚBLICAS SAUDÁVEIS}

A promoção da saúde vai além dos cuidados de saúde. Ela coloca a saúde na agenda de prioridades dos políticos e dirigentes em todos os níveis e setores, chamando-Ihes a atenção para as consequências que suas decisões podem ocasionar no campo da saúde e a aceitarem suas responsabilidades políticas com a saúde.

A política de promoção da saúde combina diversas abordagens complementares, que incluem legislação, medidas fiscais, taxações e mudanças organizacionais. É uma ação coordenada que aponta para a equidade em saúde, distribuição mais equitativa da renda e políticas sociais. As ações conjuntas contribuem para assegurar bens e serviços mais seguros e saudáveis, serviços públicos saudáveis e ambientes mais limpos e desfrutáveis.

A política de promoção da saúde requer a identificação e a remoção de obstáculos para a adoção de políticas públicas saudáveis nos setores que não estão diretamente ligados à saúde. O objetivo maior deve ser indicar aos dirigentes e políticos que as escolhas saudáveis são as mais fáceis de realizar. 


\section{CRIANDO AMBIENTES FAVORÁVEIS}

Nossas sociedades são complexas e inter-relacionadas. Assim a saúde não pode estar separada de outras metas e objetivos. As inextricáveis ligações entre a população e seu meio-ambiente constituem a base para uma abordagem socioecológica da saúde. O princípio geral orientador para o mundo, as nações, as regiões e até mesmo as comunidades é a necessidade de encorajar a ajuda recíproca - cada um a cuidar de si próprio, do outro, da comunidade e do meio-ambiente natural.

A conservação dos recursos naturais do mundo deveria ser enfatizada como uma responsabilidade global. Mudar os modos de vida, de trabalho e de lazer tem um significativo impacto sobre a saúde. Trabalho e lazer deveriam ser fontes de saúde para as pessoas. A organização social do trabalho deveria contribuir para a constituição de uma sociedade mais saudável.

A promoção da saúde gera condições de vida e trabalho seguras, estimulantes, satisfatórias e agradáveis.

O acompanhamento sistemático do impacto que as mudanças no meioambiente produzem sobre a saúde - particularmente, nas áreas de tecnologia, trabalho, produção de energia e urbanização - é essencial e deve ser seguido de ações que assegurem benefícios positivos para a saúde da população. A proteção do meio-ambiente e a conservação dos recursos naturais devem fazer parte de qualquer estratégia de promoção da saúde.

\section{REFORÇANDO A AÇÃO COMUNITÁRIA}

A promoção da saúde trabalha através de ações comunitárias concretas e efetivas no desenvolvimento das prioridades, na tomada de decisão, na definição de estratégias e na sua implementação, visando a melhoria das condições de saúde. 0 centro deste processo é o incremento do poder das comunidades - a posse e o controle dos seus próprios esforços e destino. 
O desenvolvimento das comunidades é feito sobre os recursos humanos e materiais nelas existentes para intensificar a autoajuda e o apoio social, e para desenvolver sistemas flexíveis de reforço da participação popular na direção dos assuntos de saúde. Isto requer um total e contínuo acesso à informação, às oportunidades de aprendizado para os assuntos de saúde, assim como apoio financeiro adequado.

\section{DESENVOLVENDO HABILIDADES PESSOAIS}

A promoção da saúde apoia o desenvolvimento pessoal e social através da divulgação e informação, educação para a saúde e intensificação das habilidades vitais. Com isso, aumentam as opções disponíveis para que as populações possam exercer maior controle sobre sua própria saúde e sobre o meio-ambiente, bem como fazer opções que conduzam a uma saúde melhor.

É essencial capacitar as pessoas para aprender durante toda a vida, preparando-as para as diversas fases da existência, o que inclui o enfrentamento das doenças crônicas e causas externas. Esta tarefa deve ser realizada nas escolas, nos lares, nos locais de trabalho e em outros espaços comunitários. As ações devem se realizar através de organizações educacionais, profissionais, comerciais e voluntárias, bem como pelas instituições governamentais.

\section{REORIENTAÇÃO DOS SERVIÇOS DE SAÚDE}

A responsabilidade pela promoção da saúde nos serviços de saúde deve ser compartilhada entre indivíduos, comunidade, grupos, profissionais da saúde, instituições que prestam serviços de saúde e governos. Todos devem trabalhar juntos, no sentido de criarem um sistema de saúde que contribua para a conquista de um elevado nível de saúde.

O papel do setor saúde deve mover-se, gradativamente, no sentido da promoção da saúde, além das suas responsabilidades de prover serviços clínicos e de urgência. Os serviços de saúde precisam adotar uma postura abrangente, que perceba e respeite as peculiaridades culturais. Esta postura deve apoiar as 
necessidades individuais e comunitárias para uma vida mais saudável, abrindo canais entre o setor saúde e os setores sociais, políticos, econômicos e ambientais.

A reorientação dos serviços de saúde também requer um esforço maior de pesquisa em saúde, assim como de mudanças na educação e no ensino dos profissionais da área da saúde. Isto precisa levar a uma mudança de atitude e de organização dos serviços de saúde para que focalizem as necessidades globais do indivíduo, como pessoa integral que é.

\section{VOLTADOS PARA O FUTURO}

A saúde é construída e vivida pelas pessoas dentro daquilo que fazem no seu dia-a-dia: onde elas aprendem, trabalham, divertem-se e amam. A saúde é construída pelo cuidado de cada um consigo mesmo e com os outros, pela capacidade de tomar decisões e de ter controle sobre as circunstâncias da própria vida, e pela luta para que a sociedade ofereça condições que permitam a obtenção da saúde por todos os seus membros.

Cuidado, holismo e ecologia são temas essenciais no desenvolvimento de estratégias para a promoção da saúde. Além disso, os envolvidos neste processo devem ter como guia o princípio de que em cada fase do planejamento, implementação e avaliação das atividades de promoção da saúde, homens e mulheres devem participar como parceiros iguais.

\section{COMPROMISSOS COM A PROMOÇÃO DA SAÚDE}

Os participantes desta Conferência comprometem-se a:

- atuar no campo das políticas públicas saudáveis e advogar um compromisso político claro em relação à saúde e à equidade em todos os setores;

- agir contra a produção de produtos prejudiciais à saúde, a degradação dos recursos naturais, as condições ambientais e de vida não-saudáveis e a má-nutrição; e centrar sua atenção nos novos temas da saúde pública, tais como a poluição, o trabalho perigoso e as questões da habitação e dos assentamentos rurais; 
- atuar pela diminuição do fosso existente, quanto às condições de saúde, entre diferentes sociedades e distintos grupos sociais, bem como lutar contra as desigualdades em saúde produzidas pelas regras e práticas desta mesma sociedade; - reconhecer as pessoas como o principal recurso para a saúde; apoiá-las e capacitalas para que se mantenham saudáveis a si próprias, às suas famílias e amigos, através de financiamentos e/ou outras formas de apoio; e aceitar a comunidade como porta-voz essencial em matéria de saúde, condições de vida e bem-estar;

- reorientar os serviços de saúde e os recursos disponíveis para a promoção da saúde; incentivar a participação e colaboração de outros setores, outras disciplinas e, mais importante, da própria comunidade;

- reconhecer a saúde e sua manutenção como o maior desafio e o principal investimento social dos governos; e dedicar-se ao tema da ecologia em geral e das diferentes maneiras de vida;

- a Conferência conclama a todos os interessados juntar esforços no compromisso por uma forte aliança em torno da saúde pública.

\section{POR UMA AÇÃO INTERNACIONAL}

A Conferência conclama a OMS e outras organizações internacionais para a defesa da promoção da saúde em todos os fóruns apropriados e para o apoio aos países no estabelecimento de estratégias e programas para a promoção da saúde.

A Conferência está firmemente convencida de que se as pessoas, as ONGs e organizações voluntárias, os governos, a OMS e demais organismos interessados, juntarem seus esforços na introdução e implementação de estratégias para a promoção da saúde, de acordo com os valores morais e sociais que formam a base desta Carta, a Saúde Para Todos no Ano 2000 será uma realidade

\section{CONSIDERAÇÕES FINAIS}

Desde a década de 1940, estudiosos começaram a pesquisar sobre o conceito de promoção da saúde e cada qual, com uma visão diferenciada sobre o que é saúde e sua promoção, mas que quando discutido e escrito os autores agregam as palavras 
e conceitos preocupados para que a população possa vir a ter uma boa saúde e qualidade de vida em seus vários aspectos biopsicossocial.

Nas conferências internacionais sobre promoção da saúde fica evidente as discussões que foram baseadas nos progressos alcançados com a Declaração de Alma- Ata para os Cuidados Primários em Saúde e com o documento da OMS sobre Saúde Para Todos, assim como o debate ocorrido na Assembleia Mundial da Saúde sobre as ações intersetoriais necessárias para o setor.

Com a publicação da Carta de Ottawa em 1986, abre-se o campo para as novas políticas públicas e novas ações no SUS - Sistema Único de Saúde, assim como ampliação de praças, parques, academias ao ar livre para que a população tenha seu momento de lazer e ao mesmo tempo a busca pela promoção da saúde e do bem estar gerando assim, uma melhor saúde e qualidade de vida.

Conclui-se, que os pré-requisitos na referida carta, são condições imprescindíveis e os recursos fundamentais para a saúde são: Paz - Habitação Educação - Alimentação - Renda - ecossistema estável - recursos sustentáveis justiça social e equidade. $O$ incremento nas condições de saúde requer uma base sólida nestes pré-requisitos básicos para a promoção da saúde, assim como a sinergia dos profissionais envolvidos em todos os ambientes de ação propostas. 


\section{REFERÊNCIAS}

BECKER, D. No seio da família: amamentação e promoção da saúde no Programa de Saúde da Família. [Mestrado] Fundação Oswaldo Cruz, Escola Nacional de Saúde Pública; 2001. 117 p.

BUSS, P. (org.) Promoção da Saúde e Saúde Pública. Contribuição para o debate entre as Escolas de Saúde Pública da América Latina. Rio de Janeiro: ENSP/ Fiocruz, 1998

CARVALHO, A. I. Da Saúde Pública às Políticas Saudáveis: saúde e cidadania na pós-modernidade. Ciência e Saúde Coletiva, Vol. 1; №1, Rio de Janeiro, 1996.

FERRAZ, S.T. Bases Conceituais de Promoção da Saúde - Brasília: OPS, 1996.

FERRAZ, S.T. Estratégia para adoção de "Cidades Saudáveis" no Brasil. S/local, 1993.

HARTMANN, C., LOPES, G.C.D., VIEIRA, F.S.F., SAMUEL, B.V. Trajetória Cronológica do Profissional de Educação Física. Revista Cognitionis, Rio de Janeiro, 2020.

LALONDE, M. A New Perspective on the Health of Canadians. Ottawa: Health and Welfare Canada, 1974.

LEAVELL, H; CLARK, E.G. Preventive Medicine for the Doctor in his Community. New York: MacGraw-Hill, 1965.

MACKEOWN, T. The role of Medicine: Dream, Mirage or Nemesis? London: Nuffield Provincial Hospitals Trust, 1976.

MELLO, M.V..O., BERNARDELLI JÚNIOR, R., MENOSSI, B.R.S., VIEIRA, F.S.F. Comportamento de Risoco para a saúde de estudantes da Universidade Estadual do Norte do Paraná (Brasil) - Uma proposta de intervenção online. Ciência e Saúde Coletiva, v.19, p. 159-164, 2014.

MENDES, E.V. Uma Agenda para a Saúde. Ed: Hucitec, São Paulo, 1996.

SEVERINO, A. J. Metodologia do Trabalho Científico. 24. ed. rev. e atual. São Paulo: Cortez, 2016.

SIGERIST, H. E. The University at the crossroads. New York: Henry Schumann Publishers, 1946.

TERRIS, M. Conceptos sobre Promocion de la Salud - Dualidades en la Teoria de la Salud Publica. Washington, OPAS/OMS, 1992. 
XAVIER, A.B. As Cartas das Conferências Internacionais Sobre Promoção da Saúde: uma análise das tendências ídeo-políticas. 7ํㅗㄴ Seminário da frente nacional contra a privatização da saúde. Saúde em Tempos de Retrocesso e Retirada de Direitos. Maceió, Alagoas, Brasil, 2017.

WORLD HEALTH ORGANIZATION - WHO. The Ottawa Charter for Health Promotion. Ottawa: Canadian Public Health Association, 1986 